\title{
DIFERENTES TÁTICAS DE REUTILIZAÇÃO DOS NINHOS POR TURDÍDEOS: RELATOS INICIAIS NO PARANÁ, SUL DO BRASIL
}

\section{DIFFERENT TACTICS OF NEST REUSE FOR THRUSHES: INITIAL REPORTS ON PARANÁ, SOUTHERN BRAZIL}

\author{
Huilquer Francisco Vogel \\ Universidade Estadual do Paraná, Campus de União da Vitória, Colegiado de Ciências \\ Biológicas. E-mail: huilquer@hotmail.com \\ Data de recebimento: $31 / 07 / 2013$ \\ Data da aprovação: 10/09/2013
}

\section{RESUMO}

O estudo de táticas de reutilização de ninhos por aves no Brasil ainda é discreto e pontual, necessitando de um maior aporte de informações. Neste contexto, foram investigados os mecanismos de reutilização de ninhos por aves da família Turdidae, em três locais no estado do Paraná: municípios de Maringá, Guarapuava e Dois Vizinhos. Foram ocasionalmente monitorados nove ninhos de três espécies, a saber: Turdus amaurochalinus $(\mathrm{n}=1)$, Turdus leucomelas $(\mathrm{n}=3)$ e Turdus rufiventris $(\mathrm{n}=$ 5), durante o período da nidificação entre as temporadas reprodutivas de janeiro de 2009 até janeiro de 2013. As táticas registradas neste trabalho foram: (a) reutilização inter e intraespecífica do ninho; (b) reutilização do ninho inter e intratemporadas reprodutivas; (c) reutilização do material do ninho inter e intratemporadas reprodutivas; e (d) construção de ninhos múltiplos inter e intratemporadas reprodutivas. Estes dados corroboram estudos anteriores e demonstram o amplo repertório comportamental de sabiás na reutilização do ninho. Novos estudos devem ser conduzidos, buscando avaliar o papel da reutilização do ninho no sucesso reprodutivo destas espécies.

Palavras-chave: Ecologia reprodutiva. Aves neotropicais. Reprodução de aves.

\begin{abstract}
Studies on tactics of nest reuse by birds in Brazil are still punctual and discrete, and more information on the subject is required. In this way, were investigated the mechanisms of nest reuse by birds of the family Turdidae in three sites in the State of Paraná, Brazil. Were monitored nine nests of three species, namely: Turdus amaurochalinus $(\mathrm{n}=1)$, Turdus leucomelas $(\mathrm{n}=3)$ and Turdus rufiventris $(\mathrm{n}=5)$ during the nesting period between the breeding seasons of January 2009 up to January 2013. The tactics observed in this study were: (a) intra and inter-specific nest reutilization; (b) within and between breeding seasons nest reuse; (c) reuse of nest materials between and within breeding seasons; and (d) construction of multiple nests between and within breeding seasons. These data corroborate former studies and demonstrate the wide behavioral range of thrushes regarding nest reuse.
\end{abstract}

Keywords: Reproductive ecology. Neotropical birds. Breeding birds. 


\section{Introdução}

Existe um aumento do gasto energético para a maioria das aves durante a reprodução. Em função disto ocorrem diferentes táticas reprodutivas destinadas à maximização do sucesso reprodutivo, com a diminuição de gastos energéticos (ALCOCK, 2005). Neste sentido, a reutilização parcial ou total do ninho é uma importante tática para aumentar o sucesso reprodutivo (FRIESEN et al., 1999; HAFSTAD et al., 2005).

Algumas hipóteses inferem que a reutilização do ninho pode estar relacionada com a proteção contra a predação, ou, ainda, pode servir como indicação de um bom sítio reprodutivo. Contudo, o mais provável é que a principal função da reutilização de ninhos seja a de proporcionar economia de tempo e energia, muito embora estes fatores possam atuar de modo complementar (WATTS, 1987). A reutilização do mesmo ninho ou mesmos locais de reprodução é bem documentada na literatura e, na maioria, enfatiza-se a função ecológica da reutilização dos ninhos (e.g., ANDREWS; SULLIVAN, 1996; HAFSTAD et al., 2005; BARNARD; CAMPBELL, 2007; MARINI et al., 2007; WIEBE et al., 2007). Existem informações disponíveis acerca da reutilização de ninhos por pequenos Passeriformes não coloniais, dentre os quais podemos citar as famílias de Aves Turdidae, Tyrannidae e Trochilidae (CAVITT et al., 1999; AGUILAR; MARINI, 2007; TRIANA; SANDOVAL, 2011).

Especificamente sobre a família Turdidae, embora existam diversos trabalhos sobre a biologia de aves do gênero Turdus (e.g., SAZIMA; D'ANGELO, 2011; SILVA et al., 2011; ALMEIDA; CÂMARA, 2012), estes, em sua maioria, não levam em consideração aspectos comportamentais e reprodutivos de qualquer aspecto sobre o papel da reutilização de ninhos, sendo que alguns poucos registros são mencionados para o Brasil por Marini et al. (2007).

Este gênero de aves é indicado para estudos de monitoramento de ninhos devido à abundância dos indivíduos em muitas áreas (VOGEL, 2012), sendo também ressaltada a facilidade de localização e durabilidade dos ninhos (CAVITT et al., 1999). Estes atributos permitem a execução de trabalhos como, por exemplo, a manipulação de ovos e a remoção de ninhos (KURUCZ et al., 2010). Evidências recentes sugerem a necessidade de se conhecerem aspectos pertinentes à história natural destes organismos (VOGEL et al., 2013).

Diante do contexto, o objetivo deste trabalho foi descrever algumas das táticas empregadas por aves do gênero Turdus (Turdidae) na reutilização de ninhos em hábitats do Sul do Brasil, contribuindo para o entendimento do repleto repertório comportamental do gênero.

\section{Materiais e Métodos}

\section{Locais, espécies e procedimentos}

O trabalho foi realizado entre janeiro de 2009 e janeiro de 2013. Foram monitorados nove ninhos de três espécies de sabiás (Tabela 1). Os ninhos estão distribuídos em três municípios paranaenses: Maringá, Guarapuava e Dois Vizinhos.

Em Maringá o clima é do tipo $C f a$ : subtropical úmido mesotérmico, com verão quente, sem estação seca definida; a temperatura média anual é de $20^{\circ} \mathrm{C}$, com média anual de precipitação em cerca de 1500 mm. A altitude média é de ca. $550 \mathrm{~m}$ e a vegetação é do tipo Estacional Semidecidual (MAACK, 1981; GALINA; GIMENES, 2006). Foi monitorado o ninho [1], situado em uma edificação na Universidade Estadual de Maringá.

Cinco ninhos [2-6] foram monitorados em Guarapuava, onde o clima é do tipo $C f b$ : temperado úmido com verão quente; temperatura média de $17^{\circ} \mathrm{C}$, com média anual de $2000 \mathrm{~mm}$ de precipitação. A altitude média é de ca. $1045 \mathrm{~m}$ e com vegetação do tipo Ombrófila Mista (MAACK, 1981; THOMAZ; VESTENA, 2003). Os ninhos estavam distribuídos em dois sítios de estudo no campus da Universidade Estadual do Centro-Oeste (sítios B e C). Outros três ninhos encontravam-se no Parque Municipal das Araucárias, sendo o sítio D caracterizado por ambiente de bosque com edificações, enquanto o sítio E estava situado num ambiente florestal.

Três ninhos [7-9] foram monitorados em Dois Vizinhos, onde o clima é transição entre $C f a$ e $C f b$; com temperatura média de $20^{\circ} \mathrm{C}$ e precipitação de $\approx 2000 \mathrm{~mm}$, com vegetação ecotonal entre Estacional Semidecidual e Ombrófila Mista (PIGOSSO et al., 2009). Neste local foram demarcados os sítios: F, num ambiente florestal; e $\mathrm{G}$, num ambiente em regeneração florestal, ambos inseridos no campus da Universidade Tecnológica Federal do Paraná. 
Foram realizadas visitas não periódicas aos locais (Tabela 1), durante o período considerado reprodutivo para a maioria das espécies da família, compreendido de setembro a fevereiro para o Sul do Brasil (CLEMENT, 2000). Sempre que uma nova tática de reutilização de ninho por turdídeos foi regis- trada, foram anotadas datas, coordenadas geográficas, tipo de processo envolvido e planta suporte do ninho (como em HAFSTAD et al, 2005). Nos sítios D e E ocorreram anilhamentos durante capturas realizadas entre 2008 e 2009 (VOGEL et al., 2012).

Tabela 1 - Identificação dos ninhos monitorados, com especificações sobre datas em que ocorreram as nidificações e observações de monitoramento.

\begin{tabular}{|c|c|c|c|c|c|}
\hline Ninhos e espécies & Sítio & Coordenadas & Mês & Ano & $\begin{array}{c}\text { Total de } \\
\text { Observações }\end{array}$ \\
\hline \multirow{2}{*}{ 1) T. leucomelas } & \multirow{2}{*}{ A } & $23^{\circ} 24^{\prime} 12,80^{\prime \prime} \mathrm{S}$ & Outubro & 2011 & 9 \\
\hline & & $51^{\circ} 56^{\prime} 19,17^{\prime \prime} \mathrm{W}$ & Outubro & 2012 & 2 \\
\hline \multirow{2}{*}{ 2) T. rufiventris } & \multirow{2}{*}{ B } & $25^{\circ} 21^{\prime 2} 24,31^{\prime \prime S}$ & Outubro & 2010 & 8 \\
\hline & & $51^{\circ} 28^{\prime} 3,01^{\prime \prime} \mathrm{W}$ & $* * *$ & $* * *$ & $* * *$ \\
\hline \multirow{2}{*}{ 3) T. rufiventris } & \multirow{2}{*}{$\mathrm{C}$} & $25^{\circ} 23^{\prime} 4,15^{\prime \prime} \mathrm{S}$ & Dezembro & 2012 & 8 \\
\hline & & $51^{\circ} 29^{\prime} 15,64^{\prime \prime} \mathrm{W}$ & Janeiro & 2013 & 5 \\
\hline \multirow{2}{*}{ 4) T. rufiventris } & \multirow{2}{*}{$\mathrm{D}$} & $25^{\circ} 2120,61^{\prime \prime} \mathrm{S}$ & Novembro & $2009-12$ & 12 \\
\hline & & $51^{\circ} 27^{\prime} 57,37^{\prime \prime} \mathrm{W}$ & $* * *$ & $* * *$ & $* * *$ \\
\hline \multirow{2}{*}{ 5) T. rufiventris } & \multirow{2}{*}{$\mathrm{E}$} & $25^{\circ} 21^{\prime} 15,82 ” S$ & Dezembro & 2009 & 10 \\
\hline & & $51^{\circ} 27^{\prime} 52,75^{\prime \prime} \mathrm{W}$ & Janeiro & 2013 & 1 \\
\hline 6) T. rufiventris & $\mathrm{E}$ & $* * *$ & Janeiro & 2013 & 1 \\
\hline \multirow{2}{*}{ 7) T. leucomelas } & \multirow{2}{*}{$\mathrm{F}$} & $25^{\circ} 41^{\prime} 41,14 ” S$ & Outubro & 2012 & 3 \\
\hline & & $53^{\circ} 6^{\prime} 4,34^{\prime \prime} \mathrm{W}$ & $* * *$ & $* * *$ & $* * *$ \\
\hline \multirow{2}{*}{ 8) T. leucomelas } & \multirow{2}{*}{$\mathrm{F}$} & $* * *$ & Outubro & 2010 & 10 \\
\hline & & $* * *$ & $* * *$ & $* * *$ & $* * *$ \\
\hline \multirow{2}{*}{ 9) T. amaurochalinus } & \multirow{2}{*}{ G } & $25^{\circ} 41^{\prime} 40,46^{\prime \prime} \mathrm{S}$ & Outubro & 2012 & 5 \\
\hline & & $53^{\circ} 6 ’ 8,37^{\prime \prime} \mathrm{W}$ & Dezembro & 2012 & 2 \\
\hline
\end{tabular}

Figura 1 - Representação geográfica dos três locais (municípios) amostrados neste estudo. Estado do Paraná, Brasil.

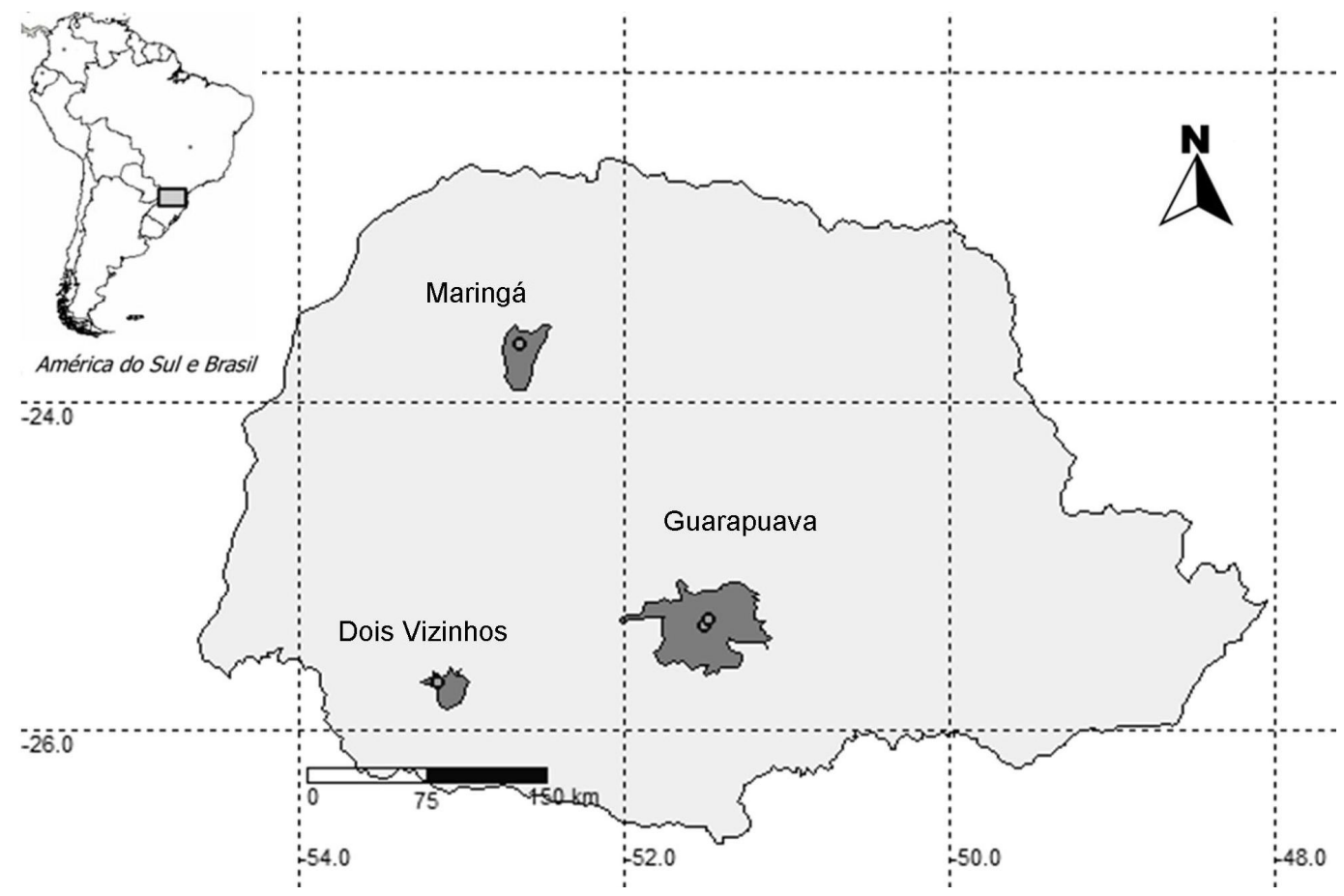




\section{Resultados}

As táticas registradas neste trabalho foram: (a) reutilização inter e intraespecífica do ninho; (b) reutilização do ninho inter e intratemporadas reprodutivas; (c) reutilização do material do ninho inter e intra- temporadas reprodutivas; e (d) construção de ninhos múltiplos inter e intratemporadas reprodutivas (Tabela 2). A reutilização do ninho inteiro pode ser pela mesma espécie ou por espécies diferentes, enquanto se observa a reutilização do local ou dos materiais dos ninhos apenas de forma intraespecífica.

Tabela 2 - Síntese de casos com reporte das diferentes táticas de reutilização do ninho de turdídeos obtidos neste estudo.

\begin{tabular}{ccc}
\hline Ninho & Formas de reutilização (táticas) & O que foi reutilizado? \\
\hline $\mathbf{1}$ & Nidificações múltiplas de forma intraespecífica & Local e território \\
\hline $\mathbf{2}$ & Reutilização interespecífica & Ninho \\
\hline $\mathbf{3}$ & Nidificações múltiplas de forma intraespecífica & Local e território \\
\hline $\mathbf{4}$ & Reutilização intraespecífica & Ninho \\
\hline $\mathbf{5}$ & Nidificações múltiplas de forma intraespecífica & Local e território \\
\hline $\mathbf{6}$ & Reutilização intraespecífica & Ninho \\
\hline $\mathbf{7}$ & Nidificações múltiplas de forma intraespecífica & Local e território \\
\hline $\mathbf{8}$ & Reutilização intraespecífica & Ninho, material e território \\
\hline $\mathbf{9}$ & Nidificações múltiplas de forma intraespecífica & Local e território
\end{tabular}

\section{Detalhamento descritivo de cada caso}

1. Foi registrada a construção simultânea de três ninhos de Turdus leucomelas. Novamente, no mesmo local, um indivíduo (presume-se o mesmo do ano anterior) efetuou a construção de três ninhos durante a temporada reprodutiva seguinte.

2. Houve a nidificação em uma cerejeira (Prunus serrulata Lindl.). Após, o ninho foi utilizado por um casal da pomba avoante Zenaida auriculata, onde nidificaram por diversas ocasiões até a queda do ninho no ano seguinte.

3. Foram registradas duas nidificações consecutivas de T. rufiventris em um ninho construído sobre a planta Cotoneaster franchetii Bois. Em ambas as ocasiões, dois filhotes deixaram o ninho com sucesso.

4. Após a primeira nidificação, os indivíduos anilhados retornaram durante quatro anos consecutivos ao mesmo local, construindo a cada ano um novo ninho sobre o ninho anterior.

5. Após a construção de um ninho sobre um xaxim (Dicksonia sellowiana Hook.), eclodiram dois filhotes. Posteriormente (2009), o mesmo ninho foi encontrado com três filhotes bem emplumados. Acredita-se que, pelo menos, um dos indivíduos adultos não fosse o mesmo do casal dos anos anteriores, pois um possuía plumagem característica de um jovem adulto, com no máximo dois anos.

6. Foi encontrado um ninho de T. rufiventris sobre uma planta caída de $D$. sellowiana. Uma análise mais apurada demonstrou a existência de dois ninhos, o mais recente construído sobre um ninho anterior.

7. Um ninho abandonado de Turdus leucomelas foi removido de local por indivíduos desta mesma espécie.

8. Foi registrada a construção de um ninho de Turdus leucomelas sobre a planta Campomanesia xanthocarpa Berg. Após o término do ninho, o mesmo foi destruído pelo casal, que construiu um novo ninho utilizando o material do ninho antigo, novamente, sobre uma árvore de $C$. xanthocarpa. Outra vez, o ninho foi destruído e mudado para outro local, sendo reconstruído durante a primeira semana de dezembro sobre uma nova espécie de planta Ocotea puberula (Rich.) Nees. Somente neste último ninho houve a postura e incubação.

9. Observou-se um casal de Turdus amaurochalinus nidificando sobre a planta Vernonia polyanthes Less. Após a saída de dois filhotes do ninho, o casal efetuou a construção de um novo ninho, não utilizando material do ninho anterior, que se 
encontrava parcialmente destruído. Novamente, um segundo ninho foi construído sobre outra planta hospedeira de $V$. polyanthes, porém não ocorreu a incubação.

\section{Discussão}

A reutilização intra-anual dos ninhos por Turdus corrobora observações documentadas para Turdus grayi no Panamá, onde, em duas situações, a segunda ninhada ocorreu no mesmo local da primeira. Neste mesmo exemplo, foi possível observar a participação da mesma fêmea em duas ninhadas consecutivas (DYRCZ, 1983; ver os comentários de HAFSTAD et al., 2005). São documentadas incubações consecutivas bem sucedidas pelo sabiá Hylocichla mustelina (FRIESEN et al., 1999), e também a reutilização do ninho de H. mustelina por outras espécies (RICHMOND et al., 2007). Todos estes autores argumentam que a reutilização do ninho é motivada pelo sucesso da primeira ninhada. Também existem registros do reúso do ninho de diversas espécies de aves, por pombos oportunistas, como Zenaida spp. (BERGENT et al., 1997).

O padrão de distribuição dos territórios dos sabiás no hábitat pode indicar evidências sobre o motivo da reutilização interanual dos ninhos ou sítios reprodutivos. Territórios com tamanhos variados e com limites razoavelmente definidos são defendidos pelos casais de sabiás, sobretudo, em espécies não migratórias de turdídeos (LUDVIG et al., 1994; ALMEIDA; CÂMARA, 2012). A territorialidade pode estar relacionada de várias formas, deste modo, Davanço et al. (2012) propõem que o comportamento de parasitismo intraespecífico dos ninhos de T.leucomela pode estar relacionado com a disponibilidade limitada de territórios durante a reprodução, justificando esforços para reutilizar territórios já habitados.

Levando em consideração que os ninhos de sabiás sofrem pressão de predação por inúmeras espécies animais (KURUCZ et al. 2010), locais ideais para a reprodução de sabiás podem ser relativamente limitados dentro de um território, e através de consecutivas reproduções fracassadas, os indivíduos seriam capazes de identificar locais menos assediados por predadores dentro do território, obtendo êxito, e ali nidificando por mais temporadas. Tal hipótese pode ser parcialmente suportada através de evidências recentes, as quais sugerem que outra espécie da família Turdidae (Catharus guttatus) tem sua densidade de ninhos afetada positivamente pelo aumento do número de locais disponíveis para a nidificação (LOOS, 2012). Deste modo, locais de qualidade para a construção dos ninhos dentro do hábitat pode ser um importante e limitado recurso.

A reforma de ninhos e a utilização do material do ninho, que foi observada, também é reportada em relação a outras espécies, como Toxostoma rufum (CAVITT et al., 1999) e T. leucomelas (MARINI et al., 2007). É aceito que a reutilização do material do ninho seja uma forma de diminuir custos energéticos e maximizar tempo (CAVITT et al., 1999). Embora não tenha sido observado neste estudo, não é descartada a possibilidade de ocorrência da reutilização interespecífica do ninho entre espécies de sabiás, visto que a maior parte dos ninhos das aves desta família possuem estruturas semelhantes (SICK, 1997, GREENEY, 2010) e, portanto, poderiam ser reutilizados.

Neste trabalho observou-se que em $T$. amaurochalinus a construção de um novo ninho foi necessária devido ao comprometimento da estrutura do primeiro ninho. Algumas explicações para reutilização do ninho são fornecidas a partir de observações do padrão de reúso de ninhos ou de materiais por Hylocichla mustelina. Segundo Richmond et al. (2007), maiores taxas de reutilização dos ninhos podem estar relacionadas com anos climaticamente atípicos, quando indivíduos da espécie estão limitados pela indisponibilidade de recursos.

A construção de múltiplos ninhos é documentada para T. leucomelas (SICK, 1997). O autor menciona que a espécie poderia se "enganar" e construir vários ninhos em um mesmo local. Contudo, esta explicação parece pouco plausível e subjetiva, pois, biologicamente, as espécies sempre buscam o melhor (trade-off) ganho energético com o menor custo (ALCOCK, 2005). Outra hipótese pode melhor explicar a construção de ninhos múltiplos, sendo a espécie capaz de identificar a localização do ninho em um local pouco favorável, i.e., locais assediados por predadores ou por aves "parasitas" de ninho (ASTIÉ; LUCHESI, 2012), nidificando posteriormente em locais mais favoráveis do território. Existem, todavia, algumas evidências que sugerem o papel da acumulação de ninhos como estratégia para diminuir a predação (WATTS, 1987). 


\section{Considerações Finais}

A reutilização de ninhos é um assunto que ainda está longe de se ter esgotado. Existe a possibilidade de que indivíduos de outras espécies ou espécimes de Turdus spp., que co-ocorrem no hábitat ou que assumem um novo território ocupado anteriormente, sejam capazes de identificar sítios com menor pressão de predação dos ninhos, convergindo para locais específicos dentro de hábitats ou território. Deste modo atuariam reutilizando ou reconstruindo estruturas já existentes. Novos esforços devem ser conduzidos na tentativa de avaliar o papel da reutilização do ninho e do hábitat em relação ao sucesso reprodutivo de aves que utilizam esta tática.

\section{Agradecimentos}

Agradeço formalmente à CAPES e ao Programa de Pós-Graduação em Ecologia de Ambientes Aquáticos Continentais (PEA), pelo apoio financeiro e institucional. Agradeço à Dr ${ }^{\mathrm{a}}$. Adriana Kataoka, da Universidade Estadual do Centro-Oeste, por disponibilizar o laboratório de Ecologia e Educação Ambiental; e ao Dr. Cláudio Henrique Zawadzki e à Bióloga Mariana Carolina Teixeira pela leitura crítica do manuscrito.

\section{Referências}

AGUILAR, T. M.; MARINI, M. Â. Nest and nest-site reuse within and between breeding seasons by three neotropical flycatchers (Tyrannidae). Brailiam Journal of Biology, São Carlos, v.67, n.3, p. 537-540, 2007. DOI: <http://dx.doi. org/10.1590/S1519-69842007000300020>.

ALCOCK, J. Animal behavior: an evolutionary approach. Sunderland: Sinauer Associates, Inc. Print, 2005. p.564,

ALMEIDA, T. O.; CÂMARA, B. G. O. Área de vida e territorialidade de Turdus leucomelas (Passeriformes: Turdidae). Atualidades Ornitológicas, Ivaiporã. v.166, n.2, p.18-21, 2012.

ANDREWS, B. J.; SULLIVAN, M. Nest-site reuse in the Western Wood-Pewee. The Wilson Bulletin, Alburquerque, v. 108, n. 2, p. 378-380, 1996.

ASTIÉ, A. A.; LUCHESI, N. Reproductive success of the Creamy-bellied Thrush in a southern temperate zone. Wilson Journal of Ornithology, Nebraska, v 124, n. 1, p.136-141, 2012. Doi: http://dx.doi.org/10.1676/11-028.1

BARNARD, G. F.; CAMPBELL, R. W. Reuse of Rufous Hummingbird nest by Anna's Hummingbird in British Columbia. Wildlife Afield, Victoria, v. 4, n. 2, p. 256-259, 2007.
BERGENT, M. Nest reuse by western kingbirds. The Wilson Bulletin, Alburquerque, v. 109, n.4, 735-737, 1997.

CAVITT, E. J.; AARONT.T; MILLER, T. A. Brown thrasher nest reuse: a time saving resource, protection from searchstrategy predators, or cues for nest-site selection? The Condor, Los Angeles, v. 101, n. 4, p.859-862, 1999.

CLEMENT, P. Thrushes. Princeton, NJ: Princeton, 2002. p. 424

DAVANÇO, P. V.; LSOUZA,L. M. S.; OLIVEIRA, L. S.; FRANCISCO, M. R. Intraspecific Brood Parasitism of the Pale-breasted Thrush (Turdus leucomelas). The Wilson Journal of Ornithology, Nebraska, v. 124, n. 3, p. 611-614, 2012. DOI: <http://dx.doi.org/10.1676/11-144.1>

DYRCZ, A. Breeding ecology of the clay-coloured robin Turdus grayi in lowland Panama. Ibis, v. 125, p. 287-304.1983. DOI: <http://dx.doi.org/10.1111/j.1474919X.1983.tb03115.x>

FRIESEN, L. E.; WYATT, V. E.; CALDMAN, M. D. Nest reuse by Wood Thrushes and Rose-breasted Grosbeaks. The Wilson Bulletin, Alburquerque, v. 111, n. 1, p.132-133, 1999.

GALINA, A.; GIMENES, M. R. Riqueza, composição e distribuição espacial da comunidade de aves em um fragmento florestal urbano em Maringá, Norte do Estado do Paraná, Brasil. Acta Scientiarum. Biological Sciences, Maringá, v. 28, n. 4, p. 379-388, 2006. DOI: <http://dx.doi. org/10.4025/actascibiolsci.v28i4.172>

GREENEY, H. F. The nest, eggs, and nesting success of the Ecuadorian Thrush (Turdus maculirostris) in southwest Ecuador. Ornitología Colombiana, Bogotá, v.10, p. 38-42, 2010.

HAFSTAD, I.; STOKKE, B. G.; VIKAN, J. R.; RUTILA, J.; RØSKAFT, E.; MOKSNES, A. Within-year nest reuse in open-nesting, solitary breeding passerines. Ornis Norvegica, Trondheim, v. 28, n.1, p. 58-61, 2005.

KURUCZ, K.; KALLENBERGER, H.; SZIGETI, C.; PURGER, J. J. Survival probabilities of first and second clutches of blackbird (Turdus merula) in an urban environment. Archives of Biological Science, Belgrade, v. 62, n. 2, p. 489-493, 2010. DOI: <http://dx.doi.org/10.2298/ ABS1002489K>

LUDVIG, É.; TÖRÖK, J.; VANICSEK, L.; CSÖRGÖ, T. Territoriality and population regulation in urban Blackbirds (Turdus merula L.). Ornis Hungárica, Budapest, v, 4, n.1, p. 01-08, 1994.

LOSS, S. R. Nesting Density of Hermit Thrushes in a Remnant Invasive Earthworm-free Portion of a Wisconsin Hardwood Forest. The Wilson Journal of Ornithology, Alburquerque, v. 124, n.2, p. 375-379, 2012. DOI: <http:// dx.doi.org/10.1676/11-124.1> 
MAACK, R. Geografia física do Paraná. Rio de Janeiro: Editora José Olímpio, 1981.p. 442

MARINI, M. Â.; AGUILAR, T. M.; ANDRADE, R. D.; LEITE, L. O.; ANCIÃES, M.; CARVALHO, C. E. A.; DUCA, C. G.; MALDONADO-COELHO, M.; SEBAIO, F.; GONÇALVES, J. F. Biologia da nidificação de aves do sudeste de Minas Gerais, Brasil. Ararajuba, Rio de Janeiro, v. 15, n. 3, p. 367-376, 2007.

PIGOSSO, M.; BONFANTE, E.; FARIAS, E.; BECEGATO, V.; ONOFRE, S. B. Diagnóstico ambiental da bacia hidrográfica do rio Jirau alto - Dois Vizinhos - Paraná. Geo Ambiente On-Line, Jataí, v. 1, n.13, 2009.

RICHMOND, S.; NOL, E.; CAMPBELL, M.; BURKE, M. D. Conspecific and Interspecific Nest Reuse by Wood Thrush (Hylocichla mustelina). Northeastern Naturalist, Steuben , v. 14, n. 4, p. 629-636, 2007. DOI:<http:// dx.DOI.org/10.1656/1092-6194 (2007) 14 [629: CAINRB] 2.0.CO; $2>$

SAZIMA, I.; D’ANGELO, B. G. The Pale-breasted Thrush (Turdus leucomelas) preys on a gekkonid lizard and an anomalepidid snake. Revista Brasileira de Ornitologia, São Paulo, v. 19, n. 3, p. 450-452, 2011.

SILVA, K. V. K. A.; LÔBO-HAJDU, G.; ALVES, M. A. S. Sex determination in Turdus amaurochalinus (Passeriformes: Muscicapidae): morphometrical analysis supported by CHD gene. Revista de Biologia Tropical, San Jose, v. 59, n. 2, p. 789-794, 2011.

THOMAZ, E. L.; VESTENA, L. R. Aspectos climáticos de Guarapuava-Pr. Guarapuava: Editora Unicentro, 2003. p. 106

TRIANA E.; SANDOVAL L. Nest Reuse by the Scintillant Hummingbird (Selasphorus scintilla). The Wilson Journal of Ornithology, Alburquerque, v. 123, n.3, p. 635-638, 2011. DOI: <http://dx.doi.org/10.1676/10-181.1>

VOGEL, H. F. Thrushes: reasons for their use in animal ecology studies. SaBios, Campo Mourão, v. 7, n. 1, p. 6699, 2012.

VOGEL, H.F.; PUJALS, A.;ZAWADZKI, C. H. Selectividad de las diferentes redes de malla de niebla en la captura de zorzales ocurrida en un parque urbano en el centro-sur del Estado de Paraná, Brasil. Ambiência, Guarapuava, v. 8, n. 3, p. 931-937, 2012. DOI: <http://dx.doi.org/0.5777/1724>

VOGEL, H. F.; SILVA, J. C. B.; ZAWADZKI, C. H. CAMPOS, J. B. Research Into Brazilian Thrushes: bibliographies, species and next steps. Bioscience Journal, Uberlândia, v. 29, n. 2, p. 468-477, 2013.

WATTS, B. D. Old nest accumulation as a possible protection mechanism against search-strategy predators. Animal Behaviour, Amsterdam, v. 35, p.1566-1568, 1987.
WIEBE, K. L.; KOENIG, W. D.; MARTIN. K. Costs and benefits of nest reuse versus excavation in cavity-nesting birds. Annales Zoologica Fennici, Helsinki, v. 44, p. 209$217,2007$.

\section{Apêndice 1 - Espécies de aves citadas no texto}

Aves (Turdidae): Catharus guttatus (Pallas, 1811); Hylocichla mustelina (Gmelin, 1789); Turdus albicollis Vieillot, 1818; Turdus amaurochalinus Cabanis, 1850; Turdus grayi Bonaparte, 1838; Turdus leucomelas Vieillot, 1818 e Turdus rufiventris Vieillot, 1818. Mimidae: Toxostoma rufum (Linnaeus, 1758). Columbidae: Zenaida auriculata (Des Murs, 1847). 